\title{
Dentistry and Covid-19
}

The Corona virus disease 2019 (Covid-19) that started at Wuhan, China was declared as a pandemic by World Health Organization as the disease spread more rapidly across the countries. The mode of transmission of the virus is still studied extensively as the protective measures need to be made accordingly. The primary mode of transmission is reported as through aerosol and direct contact with the patient as well as indirect contact with the contaminated surfaces. The incubation period though studied extensively could not be predicted as it varies from a week to more than a month. In addition to it, studies have revealed that about $80 \%$ of the infected persons remain asymptomatic. All these have made Covid-19, a biggest challenge for the entire world. The disease is associated with high mortality rate more specifically with patients with already existing systemic conditions that add as comorbidities. The healthcare workers in the frontline are at highest risk in getting the infection.

Dentistry is the field of health care where aerosol generation and contamination cannot be avoided totally. Covid-19 has made a drastic impact on the dental practice. The regulatory bodies and associations are recommending guidelines, that are evolving day to day. The Personal Protective Equipment (PPE) are manufactured and marketed rampantly with varying designs and materials claiming superior functions one over the other. On the other hand, the industries have also started marketing sanitizers, dispensers, air purifiers and filters claiming to provide safe environment for practice. Whatever claims are made, the dental practice is still not safe. Currently, it looks as if the dental profession is at stake. But there are innumerable patients awaiting dental treatments, industries awaiting that are running to cater to the needs of dentists, students awaiting who dreamt of becoming dentists in future and the healthcare system of the country awaits the effective turn back.

Dental research in the form of in vivo studies, clinical trials, etc. will face a set back temporarily. The follow-up of the already initiated research will also be a challenge. Dental fraternity has to think of changing the challenges into opportunities and set goals for research in the new normal. More than the researchers of other disciplines, the concerned subject experts would know the pros and cons better and can design appropriate research strategies for the potential research thrust areas. Innovative solutions and altered treatment modalities with safety protocols could be developed and disseminated to meet the need of the Covid era.

\section{Subhash Chandra Parija Shivasakthy Manivasakan}

